\title{
Effect of cationic and anionic surfactants on the sorption and desorption of perfluorooctane sulfonate (PFOS) on natural sediments
}

\author{
Gang Pan ${ }^{\mathrm{a}, *}$, Chengxia Jia ${ }^{\mathrm{a}}$, Dongye Zhao ${ }^{\mathrm{b}}$, Chun You ${ }^{\mathrm{a}}$, Hao Chen ${ }^{\mathrm{a}}$, Guibin Jiang ${ }^{\mathrm{a}}$ \\ ${ }^{a}$ Research Center for Eco-Environmental Sciences, Chinese Academy of Sciences, 18 Shuangqing Road, Beijing 100085, China \\ ${ }^{\mathrm{b}}$ Department of Civil Engineering, Auburn University, Auburn, AL 36849, USA
}

Cationic surfactant CTAB can immobilize PFOS but anionic surfactant SDBS mobilize PFOS onto/from the sediments.

\section{A R T I C L E I N F O}

\section{Article history:}

Received 7 March 2008

Received in revised form 12 June 2008

Accepted 18 June 2008

\section{Keywords:}

Organic matter sorption

Distribution

Environmental fate

PFOS

Remediation

Surfactants

\begin{abstract}
A B S T R A C T
Sorption and desorption of PFOS at water-sediment interfaces were investigated in the presence of a cationic surfactant, cetyltrimethylammonium bromide (CTAB), and an anionic surfactant, sodium dodecylbenzene sulfonate (SDBS). CTAB remarkably enhanced the sorption of PFOS on the sediment. In contrast, the influence of SDBS to the sorption of PFOS was concentration dependent. Two contrasting factors were responsible for the phenomenon. One was the sorption of the surfactant itself to the sediment, which enhanced the sorption of PFOS. The other was the increase in solubility of PFOS caused by the adding of surfactants, which decreased the sorption of PFOS. SDBS had a much lower sorption capacity, but rather strong ability to increase the solubility of PFOS. High levels of SDBS remarkably reduced the sorption of PFOS on the sediment. These results imply that cationic and anionic surfactants may have contrast impacts on the distribution and transport of PFOS in the environment.
\end{abstract}

(c) 2008 Elsevier Ltd. All rights reserved.

\section{Introduction}

Perfluorooctane sulfonate (PFOS) has emerged as one of the most concerning contaminants in recent years for its endocrine disrupting effect and carcinogenic toxicity (Beach et al., 2006). The U.S. Environmental Protection Agency (EPA) states that PFOS combines "persistence, bioaccumulation, and toxicity properties to an extraordinary degree" (Auer, 2000). Although many studies are focusing on the distribution of PFOS in various environmental samples, it remains a great puzzle how PFOS is transported into rural areas such as the deep sea and pole areas (Giesy and Kannan, 2001). Knowledge on the transport and fate of this contaminant and novel remediation strategies are therefore of great interests in related fields.

Sorption and desorption are important processes in controlling the fate and transport of organic contaminants in the environment. Sorption reversibility is more important than sorption itself in controlling the physical/biological availability of sorbed contaminants and the effect of soil remediation (Pignatello and Xing, 1996; Pan et al., 2004). A number of factors can affect the sorption reversibility including sorbent type and properties, concentration and characteristics of sorbates, the presence of surfactant-like

\footnotetext{
* Corresponding author. Tel.: +86 10 62849686; fax: +8610 62923541 E-mail address: gpan@rcees.ac.cn (G. Pan).
}

solutes, and environmental conditions. Surfactants, which may exist widely in the environment (Ying, 2006), can strongly affect the sorption and desorption of many organic contaminants (JonesHughes and Turner, 2005). For instance, sorption of toluene and naphthalene on soils was enhanced in the presence of the cationic surfactant CTAB (Zhang and Zen, 2006), while sorption of atrazine (a less hydrophobic chemical) was inhibited by CTAB (Tao et al., 2006). The existence of an anionic surfactant, sodium dodecylbenzene sulfonate (SDBS), enhanced sorption of aniline and pnitrophenol on sediments or soils (Shen et al., 2003), but reduced the sorption of aldicarb (a more hydrophilic pesticide) on soils (Dai et al., 2001). PFOS contains both hydrophobic and hydrophilic moieties, making it different from many other POPs (persistent organic pollutants), therefore, the sorption and desorption behaviors of PFOS are expected to be strongly affected by cationic and anionic surfactants in a different way.

Surfactants are also employed for the remediation of contaminated soils. Cationic surfactants, such as hexadecyltrimethylammonium (HDTMA), are widely used as barriers to prevent the mobility of certain contaminants from a point source of pollution (Stapleton et al., 1994). In other circumstances, surfactants may increase the solubility of hydrophobic organic contaminants in water even below the critical micelle concentration (CMC) of the surfactant (Edwards et al., 1991), which facilitates the removal of organic contaminants from soils. Therefore, knowledge of sorption and desorption of PFOS in the presence of cationic and 
anionic surfactants is important not only for understanding the fate and transport mechanisms of PFOS, but also for the development of more effective remediation technologies.

The objective of this study is to investigate the effect of cationic/ anionic surfactants (CTAB and SDBS) on the sorption and desorption of PFOS on natural sediments, and to elucidate the underlying sorption-desorption mechanism related to the distribution and transport of PFOS in the environment.

\section{Materials and methods}

\subsection{Chemicals and materials}

The potassium salt of Perfluorooctane sulfonic (PFOS) (purum, $\geq 98 \%$ ) and ammonium acetate (for mass spectroscopy, $\geq 99.0 \%$ ) were purchased from Fluka (Milwaukee, WI, USA). Cetyltrimethylammonium bromide (CTAB) (Analytical reagent grade) and sodium dodecylbenzene sulfonate (SDBS) (Analytical reagent grade) were purchased from Beijing Lanyi Fine Chemical Co. Ltd (China). Physicochemical properties of PFOS, CTAB, and SDBS are listed in Table 1.

The sediment sample was collected from the Miyun Reservoir, Beijing, China. After collection, the sediment was sealed in a polyethylene bag. The sediment samples were air-dried, sieved $(2 \mathrm{~mm})$ to remove coarse fragments, and ground to pass a $0.18 \mathrm{~mm}$ ( $80 \mathrm{mesh}$ ) sieve. Salient physicochemical characteristics of the sediment were measured according to the routine methods used in this laboratory (Tao et al., 2006). pH value of sediment (1:2 sediment/water ratio) was 7.18. The organic carbon content was $0.75 \%$; cation exchange capacity (CEC) was $6.87 \mathrm{cmol} \mathrm{kg}^{-1}$; BET surface area was $21.3 \mathrm{~m}^{2} \mathrm{~g}^{-1}$; average particle size was $55 \mu \mathrm{m}$; and the zeta potential at $\mathrm{pH} 7.0$ in a $0.5 \mathrm{mmol} \mathrm{L}^{-1} \mathrm{CaCl}_{2}$ solution was $-10.6 \mathrm{mv}$.

\subsection{Batch sorption and desorption experiments}

All the sorption/desorption experiments were conducted in $50 \mathrm{~mL}$ polypropylene centrifuge tubes. Teflon caps or vessels were avoided. To determine the sorption equilibrium time, batch sorption kinetic tests were carried out at an initial PFOS concentration of $2.0 \mathrm{mg} \mathrm{L}^{-1}$ (solution volume $=45 \mathrm{~mL}$, and sediment mass $=3 \mathrm{~g}$ ) at $\mathrm{pH} 7.0$. The suspensions were shaken by a 2D-shaker at $250 \mathrm{rpm}$ in an incubator at $25 \pm 1{ }^{\circ} \mathrm{C}$. During the experiment, the solution pH was kept at $7.0 \pm 0.05$ by intermittent adjustment with dilute $\mathrm{HCl}$ or $\mathrm{NaOH}$ solutions. Time intervals were $10,20,30 \mathrm{~min}$, and 1, 2, 4, 8, 12, $24 \mathrm{~h}$. A high solid concentration was used to simulate the solid-water interactions in sediments or soils (Higgins and Luthy, 2006; Xu et al., 2006). The aqueous solution used in sorption experiments contained $0.5 \mathrm{mmol} \mathrm{L}^{-1}$ $\mathrm{CaCl}_{2}$ (Higgins and Luthy, 2006). Kinetic tests showed that the sorption of PFOS reached apparent equilibrium within $8 \mathrm{~h}$.

Duplicate sets of PP tubes were filled with $3.0 \mathrm{~g}$ of the sediment and $45 \mathrm{~mL}$ of a solution containing $0.5 \mathrm{mmol} \mathrm{L}^{-1}$ of $\mathrm{CaCl}_{2}$ and an initial PFOS concentration ranging from $0.5 \mathrm{mg} \mathrm{L}^{-1}$ to $5 \mathrm{mg} \mathrm{L}^{-1}$. A high concentration of PFOS was used in the experiment in order to reduce the detection errors. More sensitive analytical methods need to be employed to study the sorption of PFOS at low concentration range since under latter conditions solution concentration fall below the detection limit of current analytical protocols. To test the effect of the surfactants on the sorption behavior, CTAB or SDBS was added to the solutions at initial concentrations of 3.61, 18.1, and $36.1 \mathrm{mg} \mathrm{L}^{-1}$ for CTAB, and $4.34,21.7$, and $43.4 \mathrm{mg} \mathrm{L}^{-1}$ for SDBS (Zhu and Feng, 2003). The concentration level for the surfactants was far below the critical micelle concentration (CMC), which was $361 \mathrm{mg} \mathrm{L}^{-1}$ for CTAB or $433.5 \mathrm{mg} \mathrm{L}^{-1}$ for SDBS, respectively. The suspensions were shaken by a $2 \mathrm{D}$-shaker at $250 \mathrm{rpm}$ in an incubator at $25 \pm 1{ }^{\circ} \mathrm{C}$. During the experiment, the solution $\mathrm{pH}$ was kept at $7.0 \pm 0.05$ by intermittent adjustment with dilute $\mathrm{HCl}$ or $\mathrm{NaOH}$ solutions. All tests were duplicated. Parallel control tests in the absence of sediment showed that the total PFOS loss (e.g. sorption on the tube wall, bio- and/or photo-degradation) was $<5 \%$, which was neglected in the experiment. After $24 \mathrm{~h}$ of equilibration, the suspension was centrifuged at $9000 \mathrm{~g}$ for $30 \mathrm{~min}$. Then $1 \mathrm{~mL}$ of each supernatant was transferred to $2 \mathrm{~mL}$ GC vials, and analyzed for PFOS remaining in the aqueous phase. The sorbed amounts were computed from the difference of the initial and final solute concentrations.

Following the sorption equilibrium, selected samples were subjected to further desorption isotherm experiments following the successive dilution method (Zhao et al., 2002). First, $40 \mathrm{~mL}$ of the centrifuged supernatant was removed, and replaced with the same volume of a solution that contained the same background solution but free of PFOS. The mixtures were then re-equilibrated for $24 \mathrm{~h}$ under the identical conditions ( $\mathrm{pH}$, temperature, and rotating velocity) as for the sorption experiments. Upon equilibrium, the distribution of PFOS between the sediment and solution was analyzed following the same procedures as that for the sorption experiment. The dilution and re-equilibration procedures were repeated successively to yield the desorption isotherms.

\subsection{PFOS solubility in surfactant solutions}

To quantify the changes of aqueous solubility of PFOS in the presence of the surfactants, batch experiments were performed in a solution containing $0.5 \mathrm{mmol} \mathrm{L}^{-1}$ of $\mathrm{CaCl}_{2}$ and $0-50 \mathrm{mg} \mathrm{L}^{-1}$ of CTAB or SDBS in $50 \mathrm{~mL}$ centrifuge tubes. In each test, $45 \mathrm{mg}$ of PFOS was added in $50 \mathrm{~mL}$ of the solution, which exceeds the reported water solubility of $589 \mathrm{mg} \mathrm{L}^{-1}$ at $25^{\circ} \mathrm{C}$ (Beach et al., 2006), but lower than the CMC of $1076 \mathrm{mg} \mathrm{L}^{-1}$ for PFOS (Yuan et al., 2001). The tubes were shaken at $250 \mathrm{rpm}$ in dark for $48 \mathrm{~h}$ at $25 \pm 1{ }^{\circ} \mathrm{C}$, and then centrifuged at $9000 \mathrm{~g}$ for $30 \mathrm{~min}$ to separate the solid PFOS that is not dissolved. The supernatant was diluted and analyzed for the concentration of PFOS. Parallel tests in $0.5 \mathrm{mmol} \mathrm{L}^{-1} \mathrm{CaCl}_{2}$ without surfactants were conducted under the identical conditions.

\subsection{Chemical analyses}

The concentration of PFOS in water was measured using a high performance liquid chromatograph (HPLC) (Alliance 2695) interfaced with an electrospray mass spectrometer (Waters ZQ4000). Samples ( $10 \mu \mathrm{L}$ each) were injected into a Zorbax XDB C-18 column (inner diameter $\times$ length $=2.1 \times 150 \mathrm{~mm}$, particle size $=5 \mu \mathrm{m}$, Agilent, USA) by an automatic sampler. The mobile phase consisted of $10 \mathrm{mmol} \mathrm{L}^{-1}$ of ammonium acetate and acetonitrile at a molar ratio of 55:45. The capillary was held at $3.5 \mathrm{kV}$, and the source temperature was kept constant at $140^{\circ} \mathrm{C}$. The desolvation gas flow rate was $450 \mathrm{~L} \mathrm{~h}^{-1}$, and the desolvation temperature $350{ }^{\circ} \mathrm{C}$. The cone voltage was set to $60 \mathrm{v}$, and the cone gas flow was fixed at $60 \mathrm{Lh}^{-1}$. The mass spectrometer parameters were optimized to transmit the $[\mathrm{M}-\mathrm{K}]^{-}$ion for PFOS using atmospheric pressure ionization, operated in the electrospray negative ion mode. PFOS ion was monitored using selected reaction monitoring for ion 499 for

\section{Table 1}

Characteristics of PFOS, CTAB, and SDBS

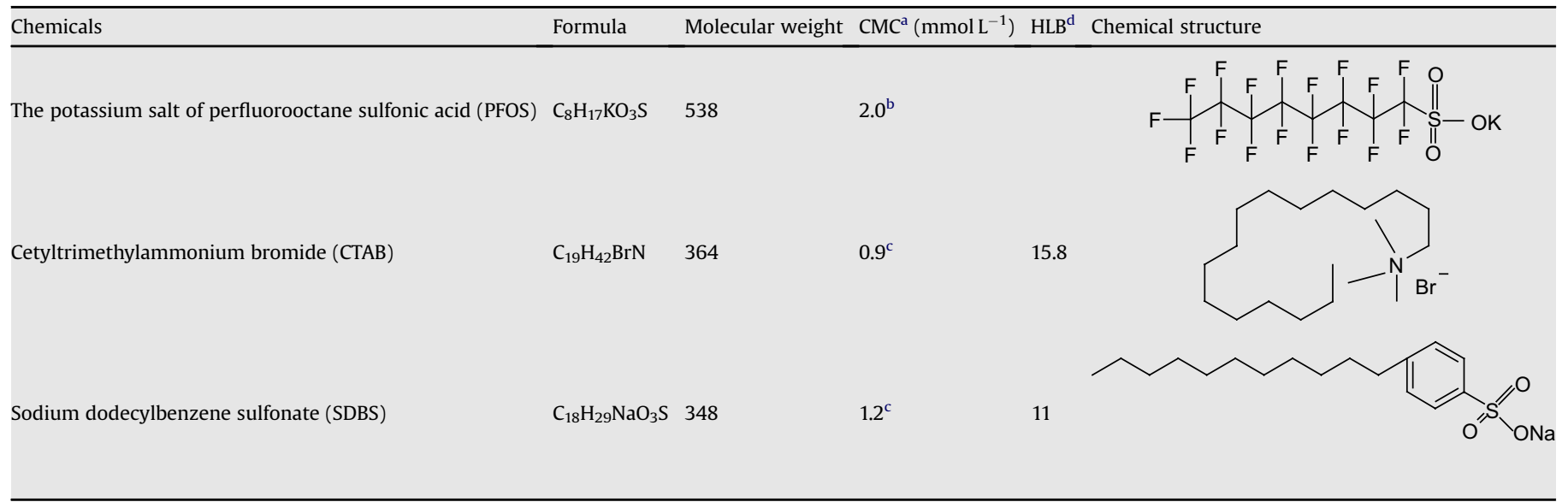

\footnotetext{
a The CMC was measured in pure water.

b Quoted from Yuan et al., 2001.

c Quoted from Zhang and Zen (2006).

d HLB (Hydrophile-Lipophile Balance Number) quoted from internet.
} 


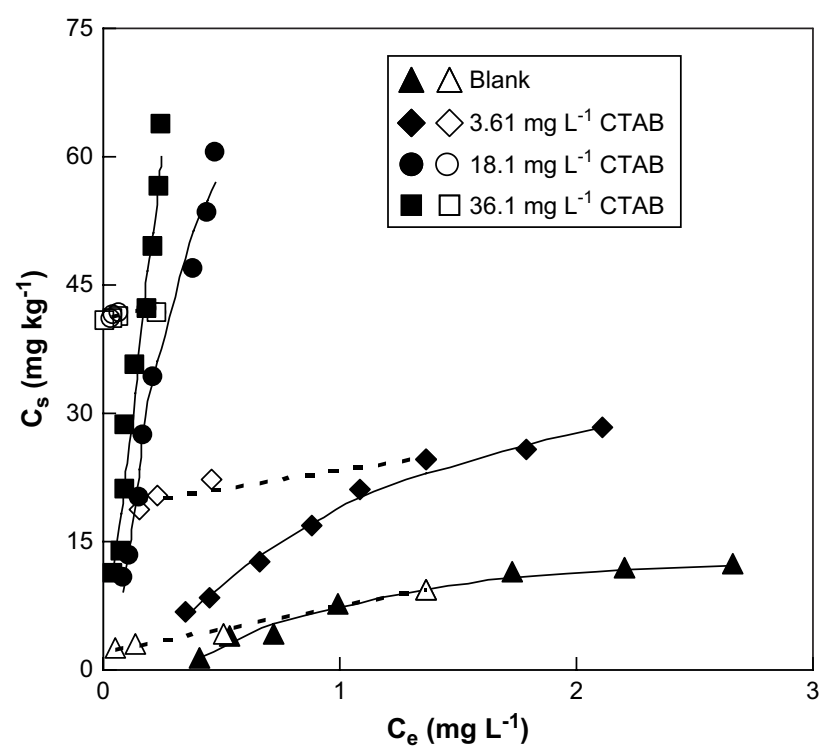

Fig. 1. Influence of the cationic surfactant $C T A B$ on the sorption and desorption isotherms of PFOS on the sediment. Solid symbols: sorption experimental data; hollow symbols: desorption experimental data; solid lines: modeled sorption isotherms; dashed lines: modeled desorption isotherms. Data given as the mean of duplicates.

quantitative determination of PFOS. SDBS concentration was determined by UV absorption at $223.6 \mathrm{~nm}$ (Rao and He, 2006). CTAB concentration was determined as described in the reference (Simon et al., 1990) using methyl orange at $418 \mathrm{~nm}$. The detection limit for PFOS used in this study was $1.5 \mu \mathrm{g} / \mathrm{L}$.

\section{Results and discussion}

\subsection{Influence of surfactants on the sorption of PFOS}

Fig. 1 shows the influence of the cationic surfactant CTAB to the sorption and desorption isotherms of PFOS on the sediment. The equilibrium data were interpreted with the Freundlich isotherm model:

$C_{\mathrm{s}}=K_{\mathrm{F}} C_{\mathrm{e}}^{n}$

where $C_{\mathrm{e}}$ is the equilibrium concentration of PFOS in solution $\left(\mathrm{mg} \mathrm{L}^{-1}\right), C_{\mathrm{s}}$ is the equilibrium concentration of PFOS $\left(\mathrm{mg} \mathrm{kg}^{-1}\right)$ in solid phase, $K_{\mathrm{F}}$ is Freundlich capacity coefficient constant, and $n$ is the Freundlich exponent reflecting the nonlinearity of the sorption isotherm. Eq. (1) can be linearized by taking the logarithm on both sides to give:

$\log C_{\mathrm{s}}=n \log C_{\mathrm{e}}+\log K_{\mathrm{F}}$
Thus, $K_{\mathrm{F}}$ and $n$ for each adsorption and desorption isotherm can be determined by fitting Eq. (2) to the respective isotherm data. Table 2 gives the model-fitted sorption and desorption parameters. However, the extrapolated $K_{\mathrm{F}}$ values from nonlinear isotherms were equilibrium concentration dependent, comparison of $K_{\mathrm{F}}$ values would bring biased results. Thus, concentration-specific distribution coefficients ( $K_{\mathrm{d}}$ values) were interpolated by Eq. (3),

$K_{\mathrm{d}}=K_{\mathrm{F}} C_{\mathrm{e}}^{n-1}$

calculated $K_{\mathrm{d}}$ values were $17.98,10.92$, and $8.80 \mathrm{~L} / \mathrm{g}$ for aqueous concentration of $0.1,0.5$ and $1.0 \mathrm{mg} / \mathrm{L}$, which is within the range of 7-45 L/g (Brooke et al., 2004; Higgins and Luthy, 2006; Johnson et al., 2007). The sediment organic carbon-normalized distribution coefficients $\left(K_{\mathrm{oc}}\right)$ were obtained from Eq. (4),

$K_{\mathrm{oc}}=K_{\mathrm{d}} / f_{\mathrm{oc}}$

Where $f_{\text {oc }}$ was fraction of organic carbon of sediments. The calculated $\log K_{\mathrm{oc}}$ values were $3.25,3.04$, and $2.94 \mathrm{~L} / \mathrm{kg}_{\mathrm{oc}}$, which was similar to the previously observed range from 2.57 to 3.1 (3M, 2000).

The presence of cationic surfactant CTAB remarkably enhanced the sorption of PFOS (Fig. 1). The $\log K_{\mathrm{F}}$ value of PFOS increased from 1.75 to $1.80,2.18$, and 2.39 when the initial CTAB increased from $0 \mathrm{mg} \mathrm{L}^{-1}$ to $3.6,18.1$, and $36.1 \mathrm{mg} \mathrm{L}^{-1}$, respectively. There was a good linear correlation between the $K_{\mathrm{F}}$ values and the initial CTAB concentration $(y=5.4 \mathrm{x}+51, r=0.998, n=4, p<0.05)$, which indicated that adding CTAB at concentrations below CMC overwhelmingly increased the sorption of PFOS.

Fig. 2 presents the sorption and desorption isotherms of PFOS in the presence of SDBS. Compared to CTAB, the effect of SDBS on the sorption of PFOS was concentration dependent. When the concentration of SDBS was lower than $4.34 \mathrm{mg} \mathrm{L}^{-1}$, SDBS slightly increased the sorption of PFOS. However, when SDBS concentration increased to 21.7 and $43.4 \mathrm{mg} \mathrm{L}^{-1}$, SDBS significantly reduced the sorption of PFOS ( $\log K_{\mathrm{F}}$ values decreased from 2.06 to 1.68 ). The reason of this concentration dependent phenomenon is analyzed in the following text (Section 3.3).

\subsection{Sorption of surfactants}

Fig. 3 shows the sorption isotherms of CTAB and SDBS on the sediment. The sediment offered a much greater sorption capacity for CTAB than SDBS. The equilibrium distribution coefficient for CTAB was 2 times greater than that of SDBS. Considering the low organic carbon content $(0.79 \%)$ of the sediment and the zeta potential value at $\mathrm{pH} 7.0(-10.6 \mathrm{mv})$, the much greater sorption capacity for CTAB was largely attributed to the electrostatic interactions between the positively charged ammonium groups $\left(\left[\left(\mathrm{CH}_{3}\right)_{3} \mathrm{NR}\right]^{+}\right.$) (the heads of $\mathrm{CTAB}$ ) and the overall negatively

Table 2

Freundlich sorption and desorption isotherm parameters, and thermodynamic index of irreversibility (TII)

\begin{tabular}{|c|c|c|c|c|c|c|c|c|c|c|}
\hline \multirow[t]{2}{*}{ Surfactants (mg L $\left.{ }^{-1}\right)$} & & \multicolumn{4}{|l|}{ Sorption } & \multicolumn{4}{|l|}{ Desorption } & \multirow[t]{2}{*}{ TII } \\
\hline & & $\log K_{\mathrm{F}}^{\mathrm{a}}$ & $n$ & $R^{2}$ & $N^{\mathrm{d}}$ & $\log K_{\mathrm{F}}$ & $n$ & $R^{2}$ & $N$ & \\
\hline Control & & $1.75 \pm 0.33^{b}$ & $0.69 \pm 0.10^{c}$ & 0.88 & 8 & $2.70 \pm 0.24^{\mathrm{b}}$ & $0.38 \pm 0.10^{c}$ & 0.88 & 4 & 0.27 \\
\hline \multirow{3}{*}{ CTAB } & 3.61 & $1.80 \pm 0.17$ & $0.81 \pm 0.06$ & 0.97 & 8 & $4.02 \pm 0.04$ & $0.12 \pm 0.02$ & 0.99 & 4 & 0.86 \\
\hline & 18.1 & $2.18 \pm 0.22$ & $0.98 \pm 0.09$ & 0.94 & 9 & $4.60 \pm 0.003$ & $0.01 \pm 0.002$ & 0.84 & 4 & 0.99 \\
\hline & 36.1 & $2.39 \pm 0.19$ & $0.99 \pm 0.09$ & 0.95 & 9 & $4.60 \pm 0.008$ & $0.01 \pm 0.004$ & 0.96 & 4 & 0.83 \\
\hline \multirow[t]{3}{*}{ SDBS } & 4.34 & $1.72 \pm 0.24$ & $0.74 \pm 0.08$ & 0.93 & 9 & $3.70 \pm 0.02$ & $0.15 \pm 0.009$ & 0.99 & 4 & 0.82 \\
\hline & 21.7 & $2.06 \pm 0.23$ & $0.57 \pm 0.07$ & 0.90 & 9 & $3.72 \pm 0.06$ & $0.08 \pm 0.02$ & 0.86 & 4 & 0.85 \\
\hline & 43.4 & $1.68 \pm 0.17$ & $0.66 \pm 0.05$ & 0.96 & 8 & $2.94 \pm 0.09$ & $0.30 \pm 0.03$ & 0.97 & 4 & 0.56 \\
\hline
\end{tabular}

${ }^{\mathrm{a}} K_{\mathrm{f}}\left(\mu \mathrm{g}^{1-\mathrm{n}} \mathrm{L}^{\mathrm{n}} \mathrm{kg}^{-1}\right)$.

b $95 \%$ Confidence interval of $\log K_{\mathrm{F}}$.

c $95 \%$ Confidence interval on $n$.

d $N$ is sample numbers. 


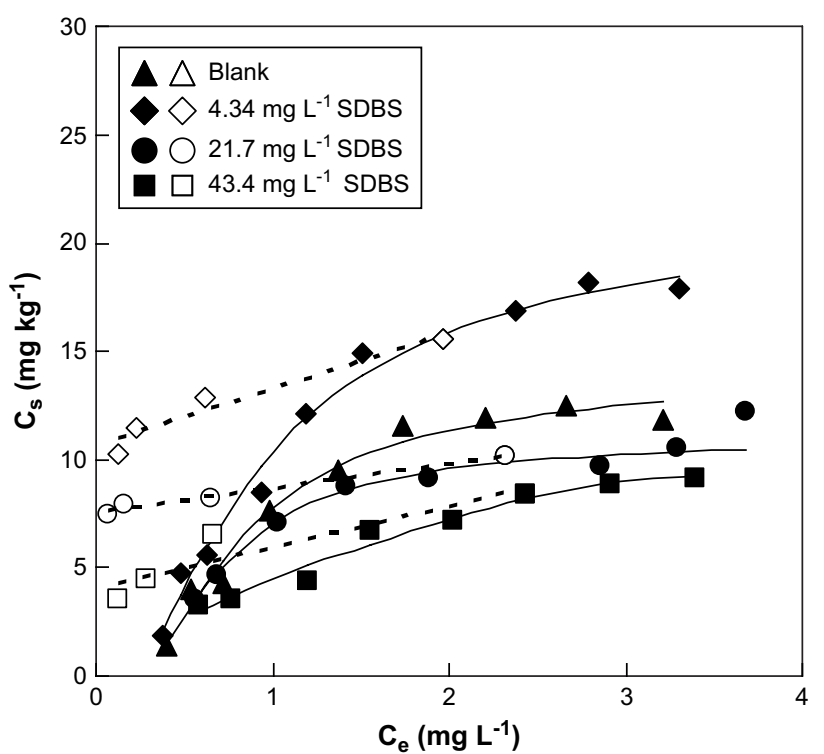

Fig. 2. Influence of the cationic surfactant SDBS on the sorption and desorption isotherms of PFOS on the sediment. Solid symbols: sorption experimental data; hollow symbols: desorption experimental data; solid lines: modeled sorption isotherms; dashed lines: modeled desorption isotherms. Data given as the mean of duplicates.

charged surface of the sediment. When CTAB was sorbed to the sediment, the hydrophobic moieties (the tails) of the surfactant formed a hydrophobic sink for hydrophobic chemicals, thereby enhancing the sorption capacity of PFOS. Sorbed surfactants can form aggregates at the solid/liquid interface at concentrations below the critical micelle concentration (Chandar et al., 1987), which have been termed hemimicelles (Gaudin and Fuerstenau, 1995) and admicelles (Harwell et al., 1985). They can exhibit hydrophobic organic chemicals partitioning capabilities because of their micellelike structures (O'Haver and Harwell, 1995). Previous studies demonstrated that hydrophobic partitioning was the dominant mechanism for sorption of PFOS to natural sediments (Jia et al., 2006; Higgins and Luthy, 2006). In this study, the sorption of PFOS became more linear with the increase of CTAB, indicating that the

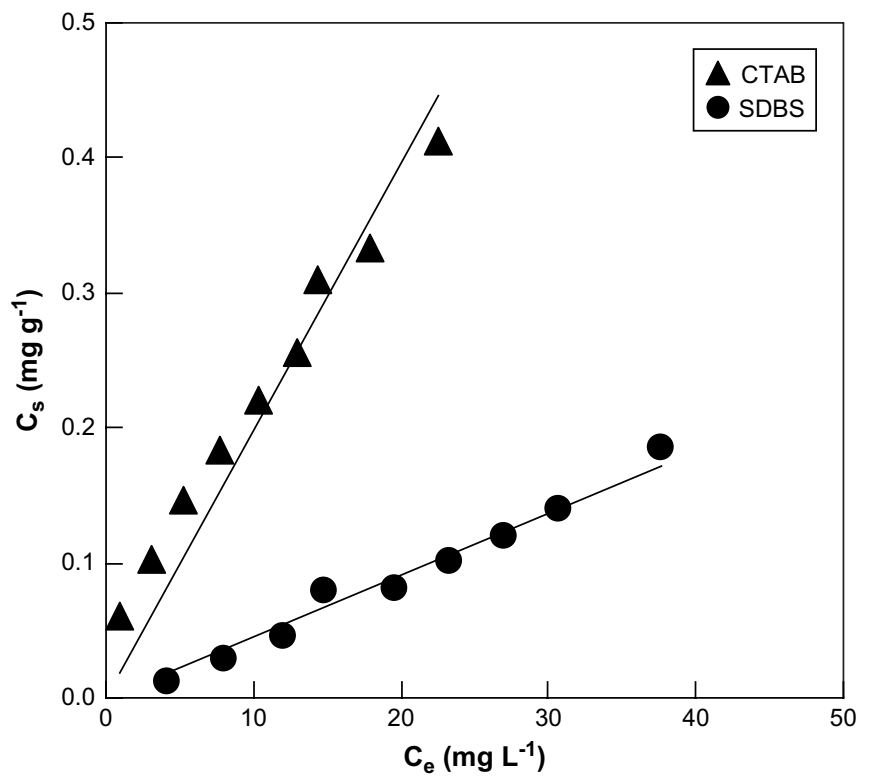

Fig. 3. Sorption isotherms of CTAB and SDBS on the sediment. Symbols: experimental data; solid lines: modeled sorption isotherms. Data given as the mean of duplicates. increased sorption of PFOS caused by the addition of surfactant may be due to the additional partition of PFOS into the sorbed CTAB.

The sorption of SDBS was lower than that of CTAB (Fig. 3). This may be largely due to the fact that SDBS is negatively charged while CTAB is positively charged (the sediment surfaces are negatively charged). Although the sorption of SDBS could increase the sorption of PFOS, one must also consider the effect of solubility changes of PFOS caused by the adding of SDBS that might reduce the sorption of PFOS. This is analyzed in the following section (3.3).

\subsection{Concentration effect of anionic surfactant CTAB on the sorption of PFOS}

Fig. 4 shows the change of solubility of PFOS in the presence of $\mathrm{CTAB}$ or SDBS. The presence of CTAB at the concentration range tested did not affect the solubility of PFOS significantly. In contrast, the aqueous solubility of PFOS was nearly doubled as the SDBS concentration increased from $5 \mathrm{mg} \mathrm{L}^{-1}$ to $40 \mathrm{mg} \mathrm{L}^{-1}$. The increased solubility of PFOS greatly diminished thermodynamic driving force for its sorption. The sorption of PFOS was progressively suppressed as the SDBS concentration increased to $21.7 \mathrm{mg} \mathrm{L}^{-1}$ and $43.4 \mathrm{mg} \mathrm{L}^{-1}$ (Fig. 2). At low concentrations of SDBS before the solubility effect became obvious, the sorption of SDBS may still be able to increase the sorption of PFOS to some extent. The two contrasting factors result in a concentration dependent effect of SDBS on the sorption of PFOS.

A previous study found that solubility of PFOS decreased with increasing ionic strength. Potassium salt of PFOS has a solubility of $370 \mathrm{mg} \mathrm{L}^{-1}$ in freshwater and $25 \mathrm{mg} \mathrm{L}^{-1}$ in filtered seawater (OECD, 2002). Solubility of PFOS decreased from $589 \mathrm{mg} \mathrm{L}^{-1}$ in pure water (Beach et al., 2006) to $304 \mathrm{mg} \mathrm{L}^{-1}$ in $0.5 \mathrm{mmol} \mathrm{L}^{-1} \mathrm{CaCl}_{2}$. The decreased solubility of PFOS in $\mathrm{CaCl}_{2}$ solution may contribute to an increase in the sorption of PFOS. This effect might exist at low levels of SDBS, but totally counteracted at high levels of SDBS when the solubility of PFOS was largely increased.

\subsection{Sorption reversibility}

Figs. 1 and 2 showed that the sorption reversibility decreased progressively as the concentration of CTAB or SDBS increased. The

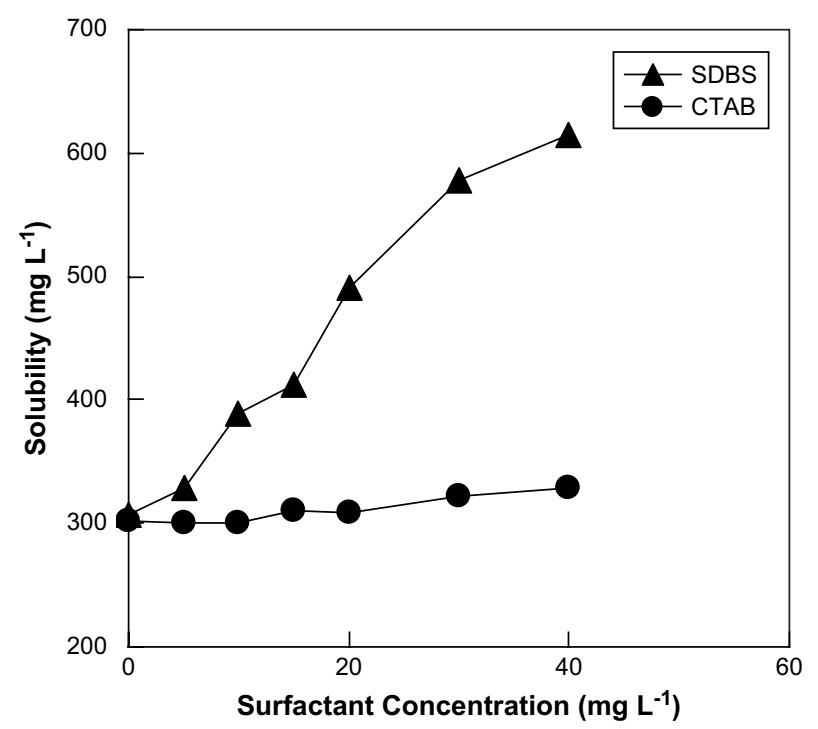

Fig. 4. Solubility of PFOS as a function of CTAB or SDBS concentration. Data given as mean of duplicates. 
degree of sorption irreversibility can be quantified using the Thermodynamic Index of Irreversibility (TII) (Sander et al., 2005):

$\mathrm{TII}=\frac{\ln C_{\mathrm{e}}^{\gamma}-\ln C_{\mathrm{e}}^{\mathrm{D}}}{\ln C_{\mathrm{e}}^{\mathrm{S}}-\ln C_{\mathrm{e}}^{\mathrm{D}}}$

where $C_{\mathrm{e}}^{\mathrm{S}}$ is the solution-phase concentration of PFOS at the sorption state $S\left(C_{\mathrm{e}}^{\mathrm{S}}, C_{\mathrm{s}}^{\mathrm{S}}\right)$ from which desorption is initiated; $C_{\mathrm{e}}^{\mathrm{D}}$ is the solution-phase concentration of PFOS at the desorption state $D\left(C_{\mathrm{e}}^{\mathrm{D}}, C_{\mathrm{s}}^{\mathrm{D}}\right) ; C_{\mathrm{e}}^{\gamma}$ is the solution-phase concentration of the hypothetical reversible desorption state $\gamma\left(C_{\mathrm{e}}^{\gamma}, C_{\mathrm{s}}^{\gamma}\right)$. $C_{\mathrm{e}}^{\mathrm{S}}$ and $C_{\mathrm{e}}^{\mathrm{D}}$ are determined from the sorption and desorption isotherms (Figs. 1 and 2 ), respectively. $C_{\mathrm{e}}^{\gamma}$ corresponds to the aqueous phase concentration on the sorption isotherm branch where the solid phase concentration of PFOS $\left(C_{\mathrm{S}}^{\gamma}\right)$ is equal to $C_{\mathrm{S}}^{\mathrm{D}}$. TII is based on the difference in free energy between the real desorption state and the hypothetical fully reversible state. The TII values lies between 0 and 1 , with 0 indicating a completely reversible system and 1 complete irreversibility.

Table 2 lists the calculated TII values for the sorption and desorption isotherms shown in Figs. 1 and 2. The sorption irreversibility (TII values) increased with the increase of CTAB. The TII values approached to 1 in the presence of $18.1 \mathrm{mg} \mathrm{L}^{-1}$ and $36.1 \mathrm{mg} \mathrm{L}^{-1}$ of $\mathrm{CTAB}$, indicating that $\mathrm{CTAB}$ not only increased sorption capacity for PFOS, but also made the sorbed PFOS highly irreversible. Previous studies also reported that sorbed nonionic surfactants such as TNP10, offered greater affinity for hydrophobic chemicals than natural sediment organic matter, and thus, inhibited desorption of organic contaminants from soils (Parker and Bielefeldt, 2003). Sorbed surfactants provide a more hydrophobic and homogeneous sink than natural soil/sediment organic matter, which typically consists of mixtures of hydrophilic and hydrophobic chemical units (Sun et al., 1995). The TII values increased with the increase of SDBS at low level range but decreased as the concentration of SDBS was higher. Unlike CTAB, at relatively high concentration levels, SDBS can largely remove the sorbed PFOS to aquatic phase.

\subsection{Implications for transport and remediation of PFOS}

The results imply that the cationic surfactant CTAB and the anionic surfactant SDBS affect the sorption of PFOS in contrasting ways. While CTAB can effectively immobilize PFOS, SDBS may mobilize PFOS in contaminated soils or sediments, e.g. limited highly polluted soils around manufacturing plants. Fig. 1 showed that nearly $98 \%$ of PFOS sorbed on the sediment remained nondesorbable in the presence of $18.1 \mathrm{mg} \mathrm{L}^{-1}$ of CTAB. This finding may be useful in understanding the distribution and transport of PFOS in the environment.

Surfactants are particularly attractive for soil remediation for their low toxicity and favorable biodegradability (Xu et al., 2006), and can be used as barriers to prevent the mobility of certain contaminants from a point source of pollution. PFOS is more watersoluble and more mobile in sediments, soils and groundwater than many hydrophobic contaminants such as PAHs. Therefore, one of the critical challenges in controlling the contamination of PFOS is to immobilize and confine its transport in the subsurface environment. For this end, our finding may be of significant practical value. Enhanced sequestration of PFOS in soils or sediments may be achieved by simply amending the contaminated media in situ with a non-harmful and/or biodegradable cationic surfactant. Cationic surfactants can be easily delivered into the contaminant source zones or down-gradient from the source by in situ percolation or injection, and immobilized the PFOS for the next cleanup treatment in the future. CTAB may serve as a simple and cost-effective remediation approach for sequestering PFOS.

\section{Conclusions}

The cationic surfactant CTAB and the anionic surfactant SDBS have contrasting impacts on the sorption and desorption of PFOS. CTAB remarkably enhanced the sorption of PFOS to the sediment. Hydrophobicity partitioning to the sorbed CTAB may be responsible for the increased sorption of PFOS. The influence of SDBS on the sorption of PFOS is concentration dependent. This behavior is regulated by two contrasting factors. One is the sorption of the surfactant which may increase the partitioning of PFOS onto the sediments/soils. The other is the possible increase in PFOS solubility caused by the adding of the surfactant, which may reduce the sorption of PFOS. This finding may be useful for understanding the cycling of PFOS in the surfactant polluted environment and for providing possible strategies for contaminated soil remediation.

\section{Acknowledgements}

The study is supported by the National Natural Science Foundation of China [20477050, 20621703] and an important-directionproject funded by the Chinese Academy of Sciences. We thank an anonymous reviewer for his/her valuable comments.

\section{References}

Auer, C., 2000. Phase out of PFOS. Internal memorandum from Charles Auer, Environmental Protection Agency (EPA). EPA Office of Pollution Prevention and Toxics Docket Number AR226-0629.

Beach, S.A., Newsted, J.L., Coady, K., Giesy, J.P., 2006. Ecotoxicological evaluation of perfluorooctanesulfonate (PFOS). Reviews of Environmental Contamination and Toxicology 186, 133-174.

Brooke, D., Footitt, A., Nwaogu, T.A., 2004. Environmental Risk Evaluation Report: Perfluorooctanesulphonate (PFOS). Prepared by Risk and Policy Analysts Ltd. for UK Environment Agency, Isis House, Howbery Park, Wallingford, UK.

Chandar, P., Somasundaran, P., Turro, N.J., 1987. Fluorescence probe studies on the structure of the adsorbed layer of dodecyl Sulfate at the alumina-water Interface. Journal of Colloid and Interface Science 117, 31-46.

Dai, S.G., Liu, G.L., Qian, Y., Chen, X.K., 2001. The sorption behavior of complex pollution system composed of aldicarb and surfactant - SDBS. Water Research 35, 2286-2290.

Edwards, D.A., Luthy, R.G., Liu, Z., 1991. Solubilization of polycyclic aromatic hydrocarbons in micellar nonionic surfactant solutions. Environmental Science \& Technology 25, 127-133.

Gaudin, A.M., Fuerstenau, D.W., 1995. Quartz flotation with anionic collectors. Transactions of AIME 202, 958-962.

Giesy, J.P., Kannan, K., 2001. Global distribution of perfluorooctane sulfonate in wildlife. Environmental Science \& Technology 35, 1339-1342.

Harwell, J.H., Hoskins, J.C., Schechter, R.S., Wade, W.H., 1985. Pseudophase separation model for surfactant adsorption: isomerically pure surfactant. Langmuir 1 , 251-262.

Higgins, C.P., Luthy, R.G., 2006. Sorption of perfluorinated surfactants on sediments. Environmental Science \& Technology 40, 7251-7256.

Jia, C.X., Pan, G., Chen, H., 2006. Sorption and desorption behavior of perfluorooctane sulfonate on the natural sediments. Chinese Journal of Environmental Science 26, 1611-1617.

Johnson, R.J., Anschutz, A.J., Smolen, J.M., Simcik, Penn, R.L., 2007. The adsorption of perfluorooctane sulfonate onto sand, clay, and iron oxide surfaces. Journal of Chemical \& Engineering Data 52, 1165-1170.

Jones-Hughes, T., Turner, A., 2005. Sorption of ionic surfactants to estuarine sediment and their influence on the sequestration of phenanthrene. Environmental Science \& Technology 39, 1688-1697.

OECD, 2002. Co-operation on Existing Chemicals - Hazard Assessment of Perfluorooctane Sulfonate and its Salts, Environment Directorate Joint Meeting of the Chemicals Committe and the Working Party on Chemicals, Pesticides and Biothechnology, Organisation for Economic Co-operation and Development, Paris.

O’Haver, J.H., Harwell, J.H., 1995. In: Sharma, R. (Ed.), Surfactant Adsorption and Surface Solubilization. ACS Symposium Series, vol. 615. American Chemical Society, Washington, pp. 49-66.

Pan, G., Qin, Y., Li, X., Hu, T., Wu, Z., Xie, Y., 2004. EXAFS Studies on adsorptiondesorption reversibility at manganese oxides-water interfaces I. Irreversible adsorption of zinc on manganite $(\gamma-\mathrm{MnOOH})$. Journal of Colloid and Interface Science 271, 28-34.

Parker, S.K., Bielefeldt, A.R., 2003. Aqueous chemistry and interactive effects on non-ionic surfactant and pentachlorophenol sorption to soils. Water Research 37, 4663-4672.

Pignatello, J.J., Xing, B.S., 1996. Mechanisms of slow sorption of organic chemicals to natural particles. Environmental Science \& Technology 30,1-11. 
Rao, P.H., He, M., 2006. Adsorption of anionic and nonionic surfactant mixtures from synthetic detergents on soils. Chemosphere 63, 1214-1221.

3M, 2000. Soil Adsorption/Desorption Study of Potassium Perfluorooctane Sulfonate (PFOS). 3M, St. Paul, MN. U.S. Environmental Protection Agency Docket AR 2261030a 030.

Sander, M., Lu, Y., Pignatello, J.J., 2005. A thermodynamically based method to quantify true sorption hysteresis. Journal of Environmental Quality 34, 1063-1072.

Shen, X., Ma, Z., Chen, P., Zhu, L., Fang, J., 2003. Effect of surfactant on sorption of polar organic compounds in sediment. Chinese Journal of Environmental Science 24, 131-135.

Simon, M.M.B., Cozar, A.D.E., Diez, L.M.P., 1990. Spectrophotometric determination of cationic surfactants in frozen and fresh squid by ion-pair formation with methyl orange. Analyst (London) 115, 337.

Stapleton, M.G., Sparks, D.L., Dentel, S.K., 1994. Sorption of pentachlorophenol to HDTMA-clay as a function of ionic strength and pH. Environmental Science \& Technology 28, 2330-2335.

Sun, S., Inskeep, W.P., Boyd, S.A., 1995. Sorption of nonionic organic compounds in soil-water systems containing a micelle-forming surfactant. Environmental Science \& Technology 29, 903-913.
Tao, Q.H., Wang, D.S., Tang, H.X., 2006. Effect of surfactants at low concentrations on the sorption of atrazine by natural sediment. Water Environment Research 78 , 653-660.

Xu, J., Yuan, X., Dai, S.G., 2006. Effect of surfactants on desorption of aldicarb from spiked soils. Chemosphere 10, 1630-1635.

Ying, G.G., 2006. Fate, behavior and effects of surfactants and their degradation products in the environmental. Environment International 32, 417-431.

Yuan, Q., Ravikrishna, R., Valsaraj, K.T., 2001. Reusable adsorbents for dilute solution separation.5: Photodegradation of organic compounds on surfactant-modified titania. Separation and Purification Technology 24, 309-318.

Zhang, J.H., Zen, J.H., 2006. Adsorption of toluene and naphthalene on Beijing soils and its influence factor. Chinese Journal of Environmental Science 27, 18891894.

Zhao, D.Y., Hunter, M., Pignatello, J.J., White, J.C., 2002. Application of the dual-mode model for predicting competitive sorption equilibria and rates of polycyclic aromatic hydrocarbons in estuarine sediment suspensions. Environmental Toxicology and Chemistry 21, 2276-2282.

Zhu, L.Z., Feng, S.L., 2003. Synergistic solubilization of polycyclic aromatic hydrocarbons by mixed anionic-nonionic surfactants. Chemosphere 53,459-467. 